\title{
Control of Laryngeal Muscle Activity in Preterm Infants $^{1}$
}

\author{
WALDEMAR A. CARLO, PHILIP C. KOSCH, EUGENE N. BRUCE, KINGMAN P. STROHL, AND \\ RICHARD J. MARTIN \\ Departments of Pediatrics and Medicine, Case Western Reserve University, Cleveland, Ohio and the \\ Departments of Physiological Sciences and Pediatrics, University of Florida, Gainesville, Florida
}

\begin{abstract}
Control of upper airway muscles, such as those in the larynx, appears important for optimizing airflow patterns during normal respiration. Electromyograms (EMGs) of the laryngeal (LAR) area and diaphragm (DIA) were recorded with esophageal and skin electrodes, respectively, in 12 unsedated sleeping preterm infants during changes in chemical and mechanical feedback. Onset of phasic inspiratory LAR EMG preceded both DIA EMG and inspiratory airflow by $70 \pm 60$ and $180 \pm 80 \mathrm{~ms}$, respectively. Inhalation of $4 \% \mathrm{CO}_{2}$ increased both peak LAR and DIA EMGs but did not alter their temporal relationships. End expiratory occlusion prolonged both LAR $(600 \pm 120$ to $930 \pm 290 \mathrm{~ms}, p<0.05)$ and DIA EMGs $(690 \pm 180$ to $940 \pm 270 \mathrm{~ms}, p<0.005)$ as well as mechanical inspiratory time. Early braking of expiratory flow was accompanied by persistence of DIA EMG into the expiratory phase, while termination of mid- to late expiratory braking was associated with onset of the LAR EMG of the subsequent inspiration. We conclude that respiratory activity of the LAR EMG is altered by both chemical and mechanoreceptor stimulation. Furthermore, simultaneous recording of LAR and DIA EMGs suggests that upper airway and chest wall muscles have different effects on expiratory flow patterns in human infants. (Pediatr Res 22: 87-91, 1987)
\end{abstract}

\section{Abbreviations}

PCA, posterior cricoarytenoid

LAR, laryngeal

EMG, electromyogram

DIA, diaphragm

Human and animal studies have documented that various muscles of the upper airway are activated in synchrony with the respiratory cycle and modulate airflow. The activity of these upper airway muscles is modulated by chemo- and mechanoreceptor afferent feedback. Electromyographic and endoscopic studies have demonstrated that one group of upper airway muscles, the intrinsic laryngeal muscles, produce marked changes in upper airway resistance by altering the position of the vocal cords (1-5). The main abductor of the vocal cords is the PCA which is active predominantly during inspiration although other laryngeal muscles (cricothyroid, thyroarytenoid, interarytenoid, and lateral

Received December 12, 1986; accepted February 13, 1987

Correspondence Waldemar A. Carlo, M.D., Department of Pediatrics, Rainbow Babies and Childrens Hospital, 2101 Adelbert Road. Cleveland, OH 44106.

Supported in part by the National Heart, Lung and Blood Institute Grants HL25830, HL-31173, and HL-28617.

${ }_{1}^{1}$ Presented in part at the American Thoracic Society, Anaheim, CA, May 1985. cricoarytenoid) are also coordinated for fine control of laryngeal resistance $(1,4,6,7)$.

Laryngeal muscle activity may be an important determinant of breathing patterns in infants. it has been proposed that retardation of expiratory flow (expiratory braking) and grunting, characteristic of neonates with respiratory distress, are accomplished by active laryngeal adduction (8). Healthy preterm and term infants are also able to control expiratory flow in order to actively maintain end expiratory lung volume above the resting lung volume $(9,10)$, although the relative contributions of laryngeal and diaphragmatic mechanisms to this regulation of lung volume are unclear. The purpose of this investigation was to determine the response of the laryngeal muscles and the diaphragm in healthy preterm infants to changes in respiratory drive and their resultant effects on airflow patterns.

\section{METHODS}

Studies were performed on 12 preterm infants free of cardiorespiratory problems at the time of study. Mean gestational age at birth was $32 \pm 2 \mathrm{wk}$ (mean \pm SD) as determined from both maternal history and physical and neurological examination. The subjects were studied at a postnatal age of $21 \pm 8$ days when their weight was $1.7 \pm 0.2 \mathrm{~kg}$. All infants were breathing room air at the time of study, although six had recovered from respiratory distress syndrome.

The LAR EMG was obtained with silver cylinder electrodes attached approximately $1.0 \mathrm{~cm}$ apart near the tip of a 5 -French esophageal catheter (11). The catheter tip was advanced 8 to 10 $\mathrm{cm}$ from the nares depending on the infant's size, and the final position adjusted to optimize the signal to noise ratio. In preliminary studies, the distance that the catheter was initially inserted was determined from postmortem studies of infants of similar size. If an electrocardiographic artefact was obtained, the catheter was pulled back slowly until it disappeared. Phasic inspiratory electromyographic activity (LAR EMG) was obtained from a very discrete area and catheter displacement of as little as a few $\mathrm{mm}$ was sufficient to lose the signal. The catheter was then secured with tape to assure a consistent signal throughout the study. The costal DIA EMG was simultaneously recorded from two adhesive surface electrodes (Medtronic Andover Medical, Lowell, MA) placed approximately $1.0 \mathrm{~cm}$ apart over the right subcostal margin between the mid and anterior axillary lines (12). Both EMGs were amplified (Coulbourn Fiber Optic Biological Preamplifiers, Lehigh Valley, PA), band pass filtered from 30 to $300 \mathrm{~Hz}$, rectified, and fed into a moving time averager with a time constant of $100 \mathrm{~ms}$.

The electrocardiographic artefact was removed by gating (SB1 EKG Blanker, CWE, Inc., Ardmore, PA). Briefly, the raw DIA EMG was full-wave rectified and compared to a reference voltage that was adjusted to be triggered by the electrocardiogram. This signal was both stored undisturbed and sent to a delay circuit. 
The delayed signal was sent to the output until an electrocardiogram was detected. At this point, the delayed signal was replaced by the undelayed signal. Hence, the electrocardiogram was removed and replaced by an adjacent portion of the DIA EMG. This process introduced a delay of the DIA EMG of approximately $60 \mathrm{~ms}$.

Airflow and mask occlusion pressures (Validyne MP 45-18, \pm $50 \mathrm{~cm} \mathrm{H} \mathrm{H}_{2} \mathrm{O}$, Northridge, $\mathrm{CA}$ ) were measured with a nasal mask pneumotachograph modified to perform occlusions (13). The pneumotachograph was linear over flow rates of 0 to 5 liter $/ \mathrm{min}$ and had a linear resistance of $8 \mathrm{~cm} \mathrm{H} \mathrm{H}_{2} \mathrm{O} / \mathrm{liter} / \mathrm{s}$ with a dead space of less than $2.5 \mathrm{ml}$. A gas flow of $5 \mathrm{liter} / \mathrm{min}$ was delivered past the mask allowing the concentration of inspired gas to be rapidly changed. Four percent $\mathrm{CO}_{2}$ in air was administered to eight infants for up to $5 \mathrm{~min}$ or until arousal occurred (from 3 to 5 min). Data from the last min of $\mathrm{CO}_{2}$ inhalation were compared to those of the control period. End tidal $\mathrm{CO}_{2}$ sampling was performed by continuous suctioning of gas from the nasal mask to the $\mathrm{CO}_{2}$ analyzer (Cavitron model PM-20NR, Anaheim, CA) at a flow rate of $60 \mathrm{ml} / \mathrm{min}$. An average of six (four to 12) and expiratory occlusions were performed in seven infants to test volume-related reflexes. Occlusions were performed for single breaths and were separated by at least $30 \mathrm{~s}$.

Peak amplitude of EMGs was quantified from the difference between baseline activity and peak phasic activity of the moving time averager on breath by breath analysis. Peak EMGs both prior to and during $\mathrm{CO}_{2}$ inhalation were quantified in arbitrary units. Mechanical inspiratory time was measured from airflow during spontaneous breathing and from mask pressure during occlusion (14). Onset, duration, postinspiratory activity, and end of the EMGs were measured from the raw signals, and when necessary the moving averager signal was used to confirm this analysis.

The protocol was approved by the Institutional Review Board and parental informed consent was obtained prior to each study. Studies were begun immediately after a feeding, although the actual data collection occurred at any time between two feedings. The infants were studied in the supine position in a semidark, quiet room and kept inside an incubator at the infants' usual

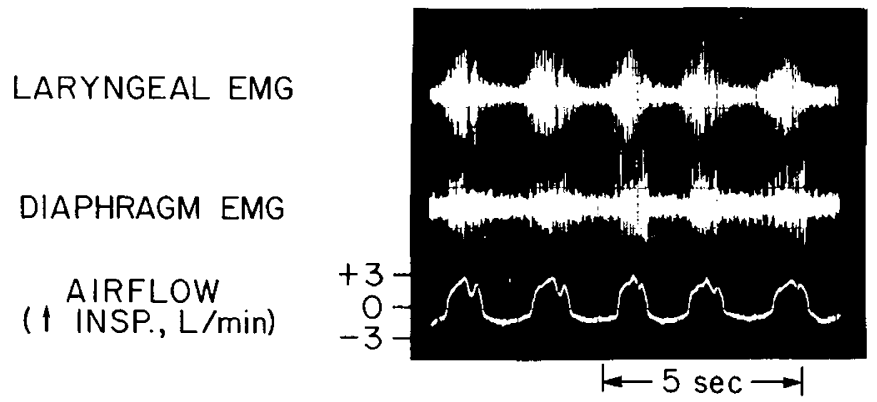

Fig. 1. LAR and DIA EMGs are shown in relation to airflow in one infant during breathing air. Inspiratory phasic LAR activity accompanies every breath. The electrocardiographic artefact on this and other figures that include the DIA EMG has been removed by gating. Notice that the late inspiratory variations in the flow are associated with changes in LAR and DIA EMGs. thermoneutral environment. Sedatives were not given to any infant and studies were performed during sleep, when respirations were regular, although classification of sleep state was not attempted in these immature infants. All data were simultaneously recorded on a polygraph. We tested the significance of the time delays between EMG events and airflow against zero using paired $t$ tests to test the null hypothesis that they occurred simultaneously. Paired $t$ tests were also used for the analysis of the rest of the data. All results are expressed as mean $\pm \mathrm{SD}$ and a $p<0.05$ was accepted for significance.

\section{RESULTS}

In each of the subjects phasic LAR and DIA EMGs could be readily recognized to occur during the inspiratory phase of the respiratory cycle (Fig. 1). As noted in Figure 1, once a phasic EMG was obtained in each infant, it was recorded virtually with every breath. Onset of LAR EMG preceded onset of DIA EMG by $70 \pm 60 \mathrm{~ms}(p<0.05)$ and inspiratory airflow by $180 \pm 80$ ms $(p<0.001$, Tablel $)$. LAR EMG ended before onset of expiratory airflow $(p<0.01)$. In eight infants DIA EMG extended into expiration for an average of $110 \pm 100 \mathrm{~ms}$. A typical example of these time delays between the EMGs and airflow is shown in Figure 2. As seen in these figures, a baseline level of activity was also present in each infant. The variability in this background activity suggests that it is predominantly tonic activity and not electrical noise. Although phasic LAR EMG occurred during inspiratory airflow in each subject, sporadic bursts of EMG from the laryngeal electrodes occasionally occurred during abrupt interruption of airflow, particularly at any time during expiration. These occasional bursts of EMG were not in synchrony with the respiratory cycle and it appeared that the obstructive episodes were associated with swallowing. Sighs were accompanied by markedly augmented LAR and DIA EMGs in each subject.

Both prior to and during $\mathrm{CO}_{2}$ inhalation, the durations of LAR and DIA EMGs were longer than inspiratory flow (Table 1). Hypercapneic stimulation increased peak LAR EMG in all eight subjects (average increase of $142 \pm 64 \%, p<0.01$ ) and

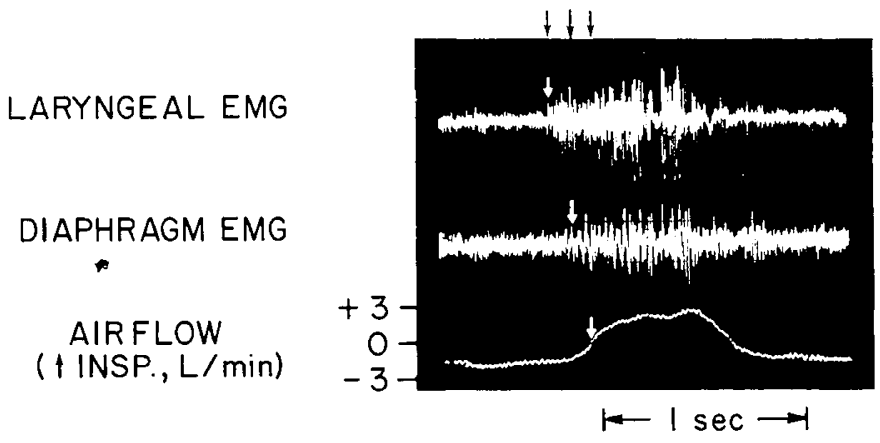

Fig. 2. Comparison of the onsets (indicated by arrows) between LAR and DIA EMGs and airflow. In this example, onset of LAR EMG precedes that of the DIA and the inspiratory airflow by approximately 50 and $100 \mathrm{~ms}$, respectively. Notice that the variation in the inspiratory flow is associated with changes in DIA EMG.

Table 1. Temporal relationships of LAR and DIA EMGs and airflow before and during $\mathrm{CO}_{2}$ inhalation (mean ?pm SD)

\begin{tabular}{|c|c|c|c|c|c|}
\hline & \multicolumn{2}{|c|}{ Difference between onsets (ms) } & \multicolumn{3}{|c|}{ Inspiratory duration (ms) } \\
\hline & $\begin{array}{l}\text { LAR to } \\
\text { DIA EMG }\end{array}$ & $\begin{array}{l}\text { LAR EMG to } \\
\text { inspiratory airflow }\end{array}$ & LAR EMG & DIA EMG & Airflow \\
\hline Control & $70 \pm 60^{*}$ & $180 \pm 80 \ddagger$ & $600 \pm 200^{*}$ & $640 \pm 240^{*}$ & $480 \pm 160$ \\
\hline $\mathrm{CO}_{2}$ & $60 \pm 40 \dagger$ & $160 \pm 50 \ddagger$ & $640 \pm 190 \dagger$ & $700 \pm 250 \dagger$ & $520 \pm 160$ \\
\hline $\mathrm{CO}_{2}$ vs control & NS & NS & NS & NS & NS \\
\hline
\end{tabular}

${ }^{*} p<0.05, \dagger p<0.005, \ddagger p<0.001$. Both EMG durations are compared to airflow. 
increased DIA EMG in seven of the eight infants studied (average increase of $50 \pm 36 \%, p<0.05$ ) while end tidal $\mathrm{CO}_{2}$ increased from $36 \pm 8$ to $44 \pm 6 \mathrm{~mm} \mathrm{Hg}$ (Fig. 3). The increases in the LAR EMG were greater than those of the DIA $(p<0.05)$. An example of the EMG responses during $\mathrm{CO}_{2}$ inhalation is shown in Figure 4. Similar to the observations during the control period, onset of LAR EMG preceded the onsets of DIA EMG $(p<$ $0.005)$ and airflow $(p<0.001)$ during hypercapnea (Table 1). The duration of EMGs and airflow were not significantly altered by hypercapnia (Table 1). $\mathrm{CO}_{2}$ inhalation did not prolong LAR EMG into expiration. Postinspiration inspiratory DIA EMG (160 $\pm 180 \mathrm{~ms}$ ) was present during $\mathrm{CO}_{2}$ inhalation in the same subjects who had it during the control period and did not appear in the other infants.

End expiratory occlusion consistently prolonged the duration of the LAR EMG $(600 \pm 120$ to $930 \pm 290 \mathrm{~ms}, p<0.05)$ and DIA EMG $(690 \pm 180$ to $940 \pm 270 \mathrm{~ms}, p<0.005)$ as well as mechanical inspiratory time $(510 \pm 130$ to $720 \pm 190 \mathrm{~ms}, p<$ 0.05 , Fig. 5). There was no consistent change in peak EMG amplitudes in response to end expiratory occlusions.

Analysis of expiratory airflow patterns during spontaneous breathing (control) and accompanying EMGs allowed us to distinguish between two patterns of braking or retarded expira- tory airflow. Retardation of early expiratory airflow was accompanied by persistence of DIA EMGs into the expiratory phase as illustrated in Figure 6. At other times expiratory airflow was retarded during mid- to late expiration followed by a rapid increase in expiratory airflow during late expiration that immediately preceded the subsequent inspiration. This flow pattern was not accompanied by consistent changes in the DIA EMG but the rapid increase in airflow toward end expiration was associated with onset of the LAR EMG of the subsequent inspiration. A typical example of this type of braking is shown in Figure 7. This pattern was seen in all subjects on at least one occasion and was associated with exhalation of up to $70 \%$ of the infants' tidal volume toward end expiration. In the absence of expiratory breaking, rapid expiratory flows commenced during early expiration and a burst of end expiratory flow did not precede the subsequent inspiration.

\section{DISCUSSION}

By means of a minimally invasive technique, we have successfully recorded electromyographic activity from the laryngeal area in healthy preterm infants. The temporal relationship of this EMG with changes in airflow and its responses to chemical and

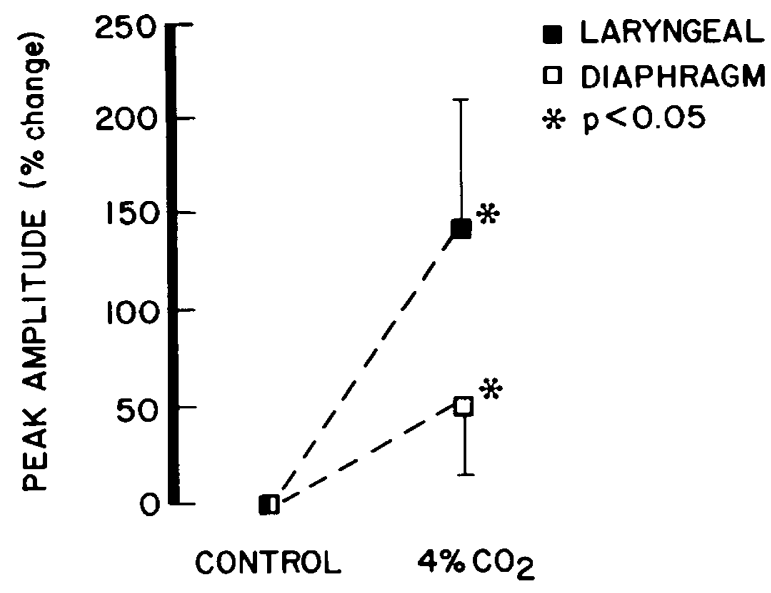

Fig. 3. Effect of $4 \% \mathrm{CO}_{2}$ inhalation on the peak amplitude of the moving time average of the LAR and DIA EMGs. Peak amplitude of LAR EMG increased more than that of the DIA.

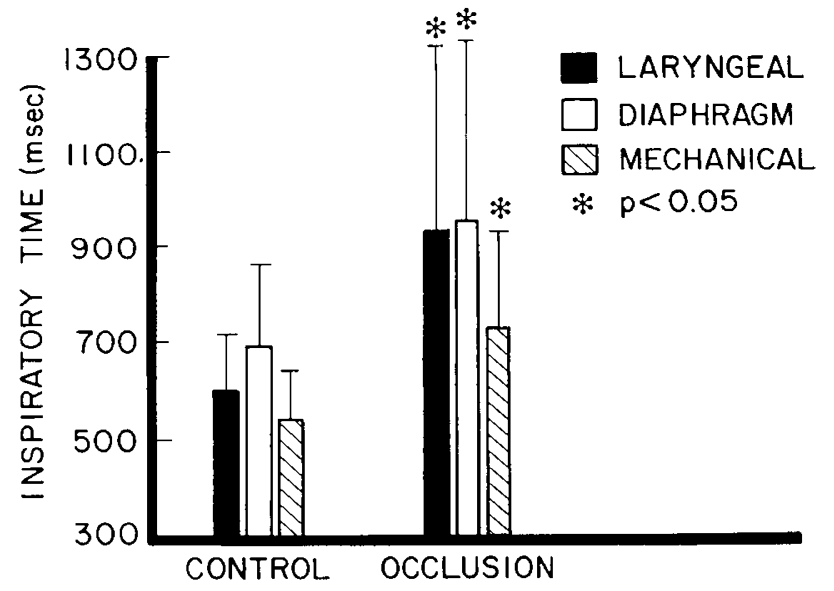

Fig. 5. Effect of end expiratory occlusion on the duration of LAR and DIA EMGs and inspiratory time. Occlusion significantly prolonged both LAR and DIA EMGs, as well as mechanical inspiratory time.

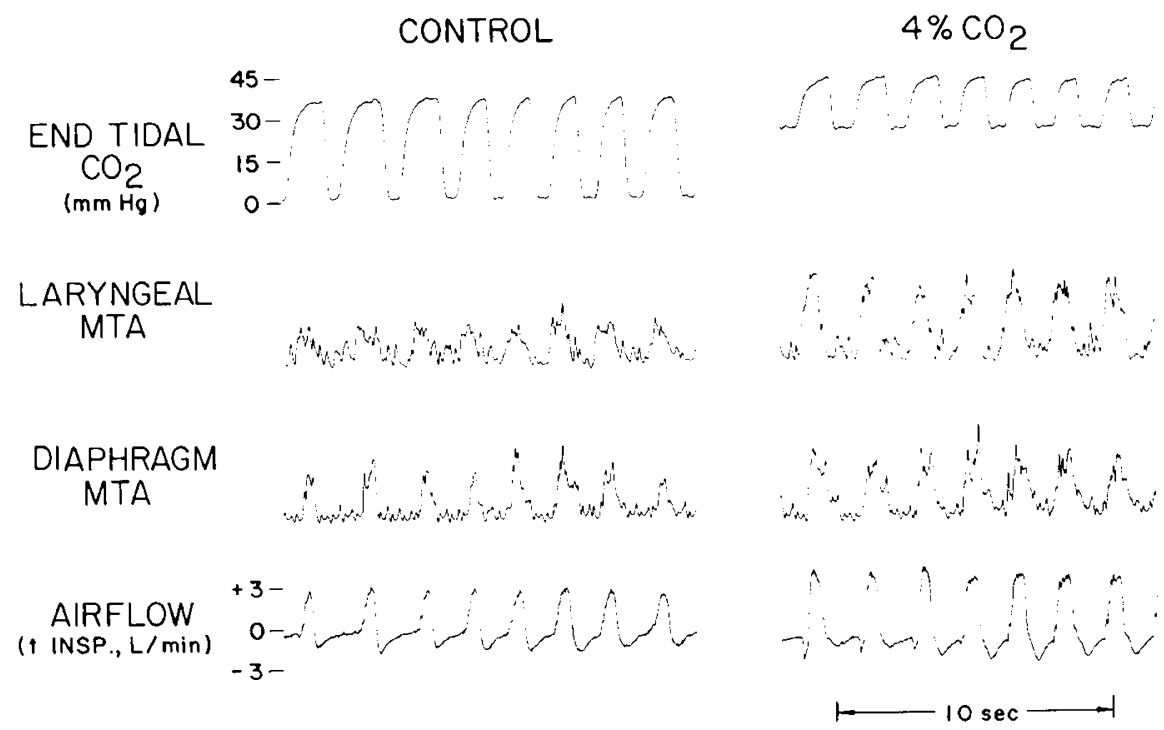

Fig. 4. Effect of $4 \% \mathrm{CO}_{2}$ inhalation on LAR and DIA EMGs moving time averages $(M T A)$ in one infant. Peak amplitude of both EMGs increased during hypercapnia but the increase in LAR EMG was greater. 


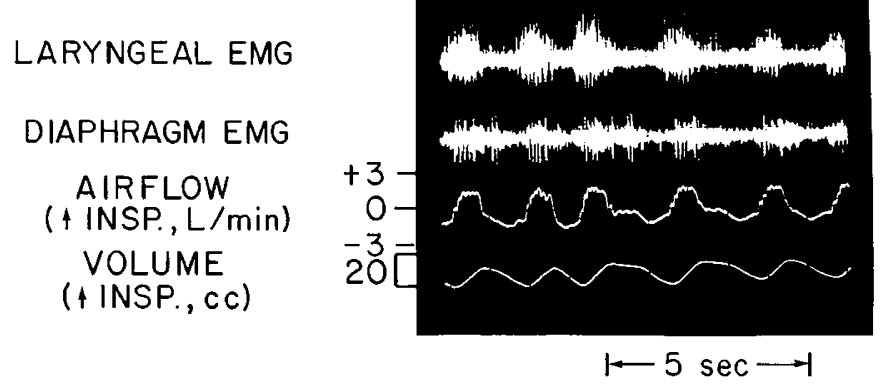

Fig. 6. Example of early expiratory braking accompanied by persistence of DIA activity beyond end inspiration. Variation in the degree of early and expiratory braking is demonstrated, being particularly prominent on the third breath.

\section{LARYNGEAL EMG}

LARYNGEAL MTA

$$
\begin{aligned}
& \text { AIRFLOW } \\
& \text { ( } \uparrow \text { INSP., L/min) } \\
& \text { VOLUME } \\
& \text { ( } \uparrow \text { INSP., cC })
\end{aligned}
$$

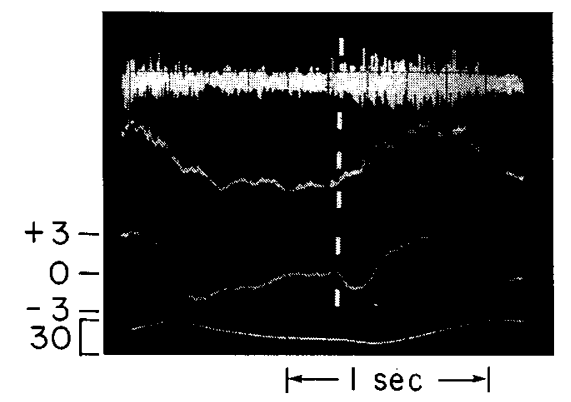

Fig. 7. Example of mid- to late expiratory braking. The rapid expiratory airflow during late expiration coincides with onset of the LAR EMG.

mechanical stimulation indicate that it represents respiratory activity from an upper airway muscle. The need for precise esophageal electrode positioning to obtain an optimal EMG suggests that the recorded activity emerges from a specific muscle or group of muscles of the laryngeal area.

Invasive recordings from muscles or nerves of the larynx have demonstrated that both inspiratory and/or expiratory activity can be observed $(6,7,15-19)$. A percutaneous needle electrode may be used to record from muscles that lie on the anterior aspect of the larynx, namely the cricothyroid and thyroarytenoid but it is technically difficult to reach the PCA, the main abductor of the vocal cords (20). Recently Guindi et al. (19) and Brancatisano et al. (21) reported the use of surface intraesophageal electrodes and documented electromyographic activity during inspiration that appeared to originate in the PCA muscles. The PCA muscles which lie on the posterior aspect of the larynx immediately anterior to the lower oropharynx, have predominantly phasic inspiratory activity and decrease inspiratory laryngeal resistance by abducting the vocal cords (1). Tonic activity of the PCA has also been observed $(7,16,18,22)$ and it is possible that persistent activity throughout expiration also reduces expiratory resistance. Other muscles of the larynx, such as the cricothyroid, may also facilitate vocal cord abduction and further decrease laryngeal resistance (1). The cricothyroid, however, is situated anterior to the vocal cords, far from our electrode position, and is thus unlikely to contribute substantially to the recorded signal. Laryngeal adductors, including the thyroarytenoid, the interarytenoid, and the lateral cricoarytenoid, are activated during expiration and typically silent during inspiration (16). Therefore, the predominantly inspiratory phasic activity recorded with our technique appears to emerge from the PCA although we cannot exclude a contribution from other intrinsic or extrinsic laryngeal muscles. The occasional bursts of activity during expiration that were associated with brief episodes of upper airway obstruction may, in turn, originate from vocal cord adductors or other muscles in the area such as the inferior pharyngeal constrictor.

The sequential pattern of respiratory muscle activation, with the onset of upper airway muscle activity preceding that of the diaphragm and subsequently airflow, has now been reported for multiple upper airway muscles in both animal and human studies including neonates (12). It has been conclusively shown that onset of PCA or recurrent laryngeal nerve activity precedes that of the diaphragm EMG or phrenic neurogram, respectively (16$18,23,24)$. Furthermore, fiber-optic visualization of the vocal cords has demonstrated that abduction of the vocal cords, which coincides with the onset of PCA EMG $(5,21)$, precedes inspiratory flow. It is possible that early activation of upper airway muscles is necessary to reduce upper airway resistance during early inspiration. Furthermore, early activation of vocal cord abductors appears to facilitate expiratory airflow just prior to inspiration. The earlier onset of LAR EMG accounts for the observation that its phasic activity was longer than inspiratory airflow. In contrast, as previously shown in newborn infants (12), DIA EMG frequently showed evidence of postinspiratory activity thus accounting for its duration beyond that of airflow. Retardation of expiratory flow during early expiration occurred in the presence of postinspiration inspiratory activity in the DIA EMG.

Once a laryngeal EMG was obtained in an infant, phasic inspiratory activity occurred with essentially every breath. This is in contrast with experience employing noninvasive or invasive EMG recordings from other upper airway muscles in infants where inspiratory activation could at times only be intermittently recorded $(12,13,25)$. These observations are in agreement with the data of Haxhiu et al. (24) in unanesthetized cats which suggest that PCA activation occurs more consistently than that of the genioglossus. The glottis represents one of the narrowest portions of the upper airway and thus activation of the PCA would be expected to be present consistently. It is also possible that the laryngeal electrodes are in closer contact to the underlying muscles than skin electrodes over the alae nasi or submental regions.

As reported with needle electrodes in animals of various species $(4,16,24,26)$ and with surface electrodes in human adults (22) hypercapneic stimulation increased the amplitude of the phasic LAR as well as DIA EMG. The relatively bigger increase of the LAR than the DIA EMG is consistent with these previous data but we are limited in our ability to determine if the increase is linear or curvilinear since only one concentration of inspired $\mathrm{CO}_{2}$ was used.

The prolongation of inspiratory activity of both LAR and DIA EMGs in response to end expiratory occlusion suggests that the activity of these muscles is modulated by volume-time related feedback. Animal studies that evaluate mechanoreceptor reflexes indicate that inspiratory inhibition of upper airway muscles may occur during normal lung inflation (27) and that these responses are, in part, vagally mediated $(4,16,23,27)$, although local upper airway receptors may also contribute (28). Since peak amplitude of the LAR EMG did not consistently increase in response to end expiratory occlusion, prolonged activation rather than increased recruitment of new fibers appears to mediate the changes that occurred during occlusion. These findings are in contrast with the consistent increase in peak amplitude of the submental EMG observed in preterm infants during occlusions (13). As noted with end expiratory occlusions in animal studies (27), it is likely that during normal lung inflation the various respiratory muscles are inhibited to different degrees. This variable response of respiratory muscles could explain the different behavior of the LAR and submental EMGs during end expiratory occlusion in preterm infants.

Expiratory braking may be accomplished by persistent activity of inspiratory muscles such as postinspiration inspiratory activity of the DIA. Braking may also be a consequence of increased expiratory resistance as occurs with active expiratory laryngeal adduction (6) or with loss of laryngeal abductor activity during 
expiration (2). Finally, a combination of some of these mechanisms may also produce expiratory braking (7). In neonatal mammals (lambs and puppies), expiratory thyroartenoid (laryngeal adductor) activity delays expiratory flow $(6,7)$. In contrast, attempts to record from various intrinsic laryngeal muscles in the newborn opossum revealed no phasic adductor activity during laryngeal closure and only demonstrated reduction of PCA activity during retardation of expiratory flow (17). Our observations seem to support the latter mechanism since the onset of phasic LAR EMG was associated with rapid expiratory airflow during late expiration suggesting that loss of PCA activity may retard expiratory flow and subsequent vocal cord abduction may counteract expiratory braking in the newborn infant. However, we cannot exclude the possibility that adductor activity could also contribute to expiratory braking in the human infant. It is also possible that the preactivation of the LAR EMG may be important in achieving a lower lung volume just before inspiration and thus a more favorable length-tension relationship for the diaphragm. The lack of other EMG recordings, particularly from the abdominal muscles, limits our ability to precisely determine the role played by other accessory muscles of respiration that may also actively modulate expiratory airflow.

Expiratory braking may have substantial effects on maintenance of lung volume, particularly in the newborn infant. The pattern of mid- to late expiratory braking with a rapid increase in expiratory flow during late expiration described in our preterm infants at $3 \mathrm{wk}$ of age has been observed to be more prominent during the first hours of life in full-term infants (29). Expiratory braking may also be of particular importance in neonates because they have increased chest wall compliance and a need to maintain end expiratory lung volume above their resting lung volumes ( 9 , 10). Persistence of DIA braking during $\mathrm{CO}_{2}$ inhalation may be a strategy used by preterm infants for active maintenance of an elevated lung volume.

In summary we have used a minimally invasive technique to record electromyographic activity of the LAR area. The location and characteristics of the phasic inspiratory LAR EMG suggest that it emerges from the PCA. Simultaneous DIA EMG recordings have also allowed further characterization of expiratory braking patterns, suggesting that LAR muscles are actively involved in determining expiratory flow patterns in preterm infants.

Acknowledgments. The authors thank Julie DiFiore for technical expertise and Nancy Kernen for preparation of the manuscript.

\section{REFERENCES}

1. Konrad HR, CC Rattenborg 1969 Combined action of laryngeal muscles. Acta Otolaryngol 67:646-649

2. Bartlett D Jr, Remmers JE, Gautier H 1973 Laryngeal regulation of respiratory airflow. Respir Physiol 18:194-204

3. Dixon M, Szereda-Przestaszewska M, Widdicombe JG, Wise JCM 1974 Studies on laryngeal calibre during stimulation of peripheral and central chemoreceptors, pneumothorax and increased respiratory loads. J Physiol 39:347363

4. Bartlett D Jr 1979 Effects of hypercapnia and hypoxia on laryngeal resistance to airflow. Respir Physiol 37:293-302

5. England SJ, Bartlett D Jr, Daubenspeck JA 1982 Influence of human vocal cord movements on airflow and resistance during eupnea. J Appl Physiol 52:773779

6. Harding P, Johnson P, McClelland ME 1980 Respiratory function of the larynx in developing sheep and the influence of sleep state. Respir Physiol 40:165-179

7. England SJ, Kent G, Stogryn HAF 1985 Laryngeal muscle and diaphragmatic activities in conscious dog pups. Respir Physiol 60:95-108

8. Harrison VC. Heese H DeV, Klein M 1968 The significance of grunting in hyaline membrane disease. Pediatrics 41:549-559

9. Mortola JP, Fisher JT, Smith B, Fox G. Weeks S 1982 Dynamics of breathing in infants. J Appl Physiol 52:1209-1215

10. Kosch PC, Stark AR 1984 Dynamic maintenance of end expiratory lung volume in full term infants. J Appl Physiol 57:1126-1133

11. Kosch PC, Hutchinson AA, Wozniak JA, Stark AR 1985 Expiratory airflow control mechanism in term infants. Fed Proc 44:1002

12. Carlo WA, Martin RJ, Abboud EF, Bruce EN, Strohl KP 1983 Effect of sleep state and hypercapnia on alae nasi and diaphragm EMGs in preterm infants. J Appl Physiol 54:1590-1596

13. Carlo WA, Miller MJ, Martin RJ 1985 Differential response of upper airway and diaphragmatic activities to airway occlusion in infants. J Appl Physiol $59: 847-852$

14. Younes MK, Remmers JE, Baker J 1978 Characteristics of inspiratory inhibition by phasic volume feedback in cats. J Appl Physiol 45:80-86

15. Murakami Y, Kirchner JA 1972 Respiratory movements of the vocal cords: an electromyographic study in the cat. Laryngoscope 82:454-467

16. Sherrey JH, Megirian D 1975 Analysis of the respiratory role of intrinsic laryngeal motoneurons of cat. Exp Neurol 49:456-465

17. Farber JP 1978 Laryngeal effects and respiration in the suckling opossum. Respir Physiol 35:189-201

18. Orem J, Lydic R 1978 Upper airway function during sleep and wakefulness: experimental studies on normal and anesthetized cats. Sleep 1:49-68

19. Guindi GM, Higenbottam TW, Payne JK 1981 A new method for laryngeal electromyography. Clin Otolaryngol 6:271-178

20. Berry H, Blair RL, Briant TDR 1977 A method of percutaneous laryngeal electromyography. J Otolaryngol 6:491-495

21. Brancatisano TP, Dodd DS, Engel LA 1984 Respiratory activity of posterior cricoarytenoid muscle and vocal cords in humans. J Appl Physiol 57:11431149

22. Brancatisano TP, Dodd DS, Engel LA 1986 Responses of the posterior cri coarytenoid and alae nasi muscle to increased chemical drive in man. Respir Physiol 64:177-189

23. Cohen MI 1975 Phrenic and recurrent laryngeal discharge patterns and the Hering-Breuer reflex. Am J Physiol 228:1489-1496

24. Haxhiu MA, van Lunteren E, Mitra J, Cherniack NS 1984 Responses to chemical stimulation of upper airway muscles and diaphragm in awake cats. J Appl Physiol 56:397-403

25. Roberts JL, Reed WR, Mathew OP, Thach BT 1986 Control of respiratory activity of geniogiossus muscle in micrognathic infants. J Appl Physio 61:1523-1533

26. Weiner D, Mitra J, Salamone J, Cherniack NS 1982 Effect of chemical stimul on nerves supplying upper airway muscles. J Appl Physiol 52:530-536

27. van Lunteren E, Strohl KP. Parker DM, Bruce EN, van de Graaff WB Cherniack NS 1984 Phasic volume-related feedback on upper airway muscle activity. J Appl Physiol 56:730-736

28. Sant'Ambrogio FB, Mathew OP, Clark WD, Sant'Ambrogio G 1985 Laryngeal influences on breathing pattern and posterior cricoarytenoid muscle activity. J Appl Physiol 58:1298-1304

29. Fisher JT, Mortola JP, Smith JB, Fox GS, Weeks S 1982 Respiration in newborns:development of the control of breathing. Am Rev Respir Dis $125: 650-657$ 